\title{
LINEAR FINITE ELEMENT METHOD IN AXISYMMETRIC MAGNETOSTATIC PROBLEMS
}

\author{
B. Lencováa) \\ Particle Optics Group \\ Department of Applied Physics \\ TU Delft, Lorentzweg 1 \\ NL-2628 CJ Delft, The Netherlands
}

\author{
M. Lenc \\ Institute of Scientific Instruments \\ Czechoslovak Academy of Sciences \\ Královopolská 147 \\ CS-61264 Brno, Czechoslovakia
}

\begin{abstract}
If there is a long iron cylinder on the axis of rotational symmetry, the FEM with linear shape function for the computation of the angular component of the vector potential yields an incorrect solution. Based on a suitable weight factor in the expression for the $z$ component of the flux density, new formulas for the coefficients of the linear equations have been derived, which give correct answers.
\end{abstract}

\section{INTRODUCTION}

In electron optics, we wish to determine the electron optical properties of lenses and other elements with high accuracy, this demands a high accuracy of field evaluation. This has contributed to the widespread appreciation of the finite element method (FEM), introduced into electron optics by Munro [1], and reviewed also in [2,3]. It was therefore an unpleasant surprise that all the computation methods used up to now for the angular component of the vector potential suffer from an unacceptable inherent error, which manifests itself strongly in the case of cylindrical high permeability rods on the axis, as in case of the BH tester of Tahir and Mulvey [4] or the pot transformer of Melissen and Simkin [5].

To overcome this problem, several solutions modifying the linear FEM were proposed. Boglietti et al [6] proposed a technique which uses weighted linear shape functions $A=r^{n}(f+g z+h r) ;$ the weighting factor $n$ changes from the value $n=0$ at the rotational axis to the value $n=1$ at the outer radial boundary. Melissen and Simkin [5] proposed to use the flux $\mathbf{\Psi}=2 \pi r A$ instead of $A$ and the linear shape functions for $\mathbf{\Psi}=f+g z+h u, u=r^{2}$

a) On leave from Inst. Sci. Instr., Brno.

Manuscript received July 8, 1991. This work was done as a part of FOM project IOP-IC-DTN 45.006.
In this paper we point out the source of the problem. Further a short description of FEM in electron optics is given, and a simple and, we believe, more suitable solution is put forward.

\section{DESCRIPTION OF THE PROBLEM}

The energy functional in cylindrical coordinates

The integration of the energy functional for $A$, namely

$$
E=2 \pi \iint\left[\frac{1}{2 \mu}\left[\left(\frac{\partial A}{\partial z}\right)^{2}+\frac{1}{r^{2}}\left(\frac{\partial A}{\partial}+\frac{A}{r}\right)^{2}\right)-J A\right] r d r d z
$$

can be performed on individual triangles of the finite element mesh with the linear shape function $A(r, z)=f+g z+h r$. Here we have, for counter clockwise notation of the triangle vertices,

$$
\begin{aligned}
f & =\frac{1}{D} \sum_{i} a_{i} A_{i}, g=\frac{1}{D} \sum_{i} b_{i} A_{i}, \\
h & =\frac{1}{D} \sum_{i} c_{i} A_{i},
\end{aligned}
$$

with $a_{i}=r_{k} z_{j} z_{k} r_{\dot{p}}, b_{i}=r_{j} r_{k}, c_{i}=z_{k} z_{\dot{p}}$ ( $(j ; j, k)$ being a cyclic permutation of $(1,2,3)$, and $D=b_{1} c_{2}-b_{2} c_{1}$ is twice the area of the triangle.

The linear shape function in the triangular finite element guarantees the continuity of the normal component of the flux density $B_{n}$ between elements containing materials with different permeability $\mu$, but not the continuity of the tangential component of the field intensity $H_{t}$. This effect is most pronounced where $B_{n}=0$ in the important parts of the magnetic circuit. One-dimensional or quasi-one-dimensional problems are the most striking examples, as revealed in [4-6], where the solution is determined by the tangential component $H_{t}$ (i.e. by $H_{z}$ ) only.

On the cylindrical boundary at $r=r_{0}$ between two materials with relative permeabilities $\boldsymbol{\mu}_{1}$ and $\boldsymbol{\mu}_{2}$, the local error of $A$ 
can be evaluated from the known analytical solution in a region with constant $H_{z}$ at the mesh points $i=1$ and $i=2$ above and below the mesh point at $r=r_{0}$, namely

$$
r_{i} A_{i}=r_{0} A_{0}+\mu_{i} H_{z}\left(r_{i}-r_{0}\right) r_{i} / 2
$$

\section{Expression of coefficients of FEM equations}

The expression of coefficients of FEM equations depends on the variant of the finite element method, particularly on the choice of mesh, and on the method chosen for the integration of (1). The meshes used in electron optics are made of quadrilateral elements. The mesh is topologically equivalent to a square mesh. The geometry of the axially symmetric lens is specified with quadrilaterals of general shape, defined with the help of a coarse mesh of horizontal and vertical lines. A smooth fine mesh with variable step size is made with the
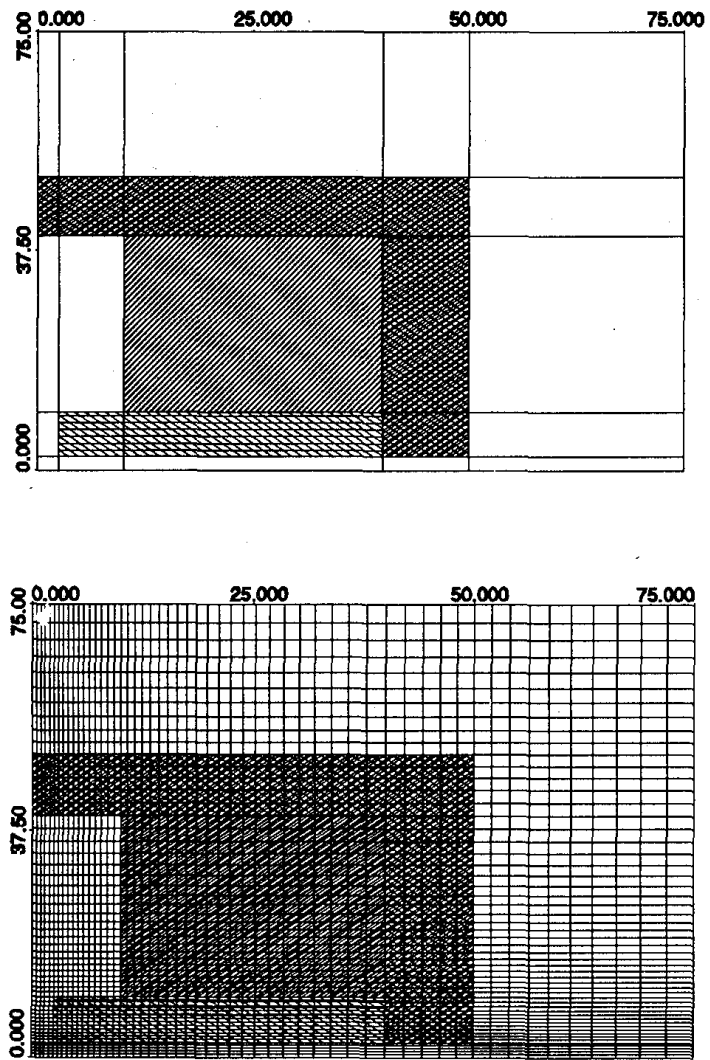

Fig. 1. Coarse and fine mesh in a magnetic lens with long polepieces. The $z$ axis is drawn horizontal; the plane at $z=0 \mathrm{~mm}$ is a symmetry plane. The gap between the polepieces and the diameter of the lens bore are $5 \mathrm{~mm}$. Fine mesh is used with 55 points in each direction. help of an automeshing procedure, built into an interactive program for input of data and display of results. This is illustrated in Fig. 1 on the coarse and fine mesh used later for the test computations.

The fine quadrilateral mesh, defined above, is for the computation of coefficients subdivided into two triangles; mostly an average from both subdivisions are used. A different expression for the coefficients of the resulting linear equations can be obtained, depending on which integration method is used either for $B_{z}=(1 / r) \partial(r A) / \partial$, the $z$ component of the flux density, or for $B_{z}{ }^{2}$ in (1). Munro originally used an average value of $B_{z}$ in the triangle.

In [3] we presented three methods of integrating eq. (1), in particular the critical term $r B_{z}^{2}$, which provide different expressions for the coefficients of the FEM equations, all of which have the same order of numerical error $O\left(h^{2}\right)$ see Fig. 2. The first method, using the gravity center of the triangle, provides identical expressions to those of Munro [1]. The second method takes the integrand values at the vertices of the triangle, and gives, for rectangular meshes, exactly the coefficients of the five point finite difference method. The third method, based on using the values at the midpoints of the triangle sides, gives mostly the best accuracy and it is not sensitive to the change in the mesh step; it is thus the most suitable one for the computation of coefficients, although it requires about $20 \%$ increase of the computation effort.

Unfortunately, all three methods have the same behavior of the local error on the cylindrical boundary in the basically one dimensional situation, e.g.

$$
A_{0}^{F E M}-A_{0}^{a \text { al }}=\frac{\rho_{1} \rho_{2}}{6 r_{0}^{3}} \frac{\rho_{1} \boldsymbol{\mu}_{2}-\rho_{2} \boldsymbol{\mu}_{1}}{\boldsymbol{\mu}_{1}+\boldsymbol{\mu}_{2}} A_{0} \text {, }
$$


Fig. 2. Triangles used for the integration of eq. (1). Squares denote the points where the function value is evaluated: in the left triangle the value of the energy functional is taken at the center of the triangle, in the right triangle at the mid points of the sides. 
with $\rho_{i}=\left|r_{i}-r_{0}\right|$. Eq. (4) was obtained by substituting the analytical solution (3) into the expression for coefficients of FEM equations for the last computation method for coefficients. The error is proportional to $A_{0}$, and thus it reaches quite a large value with a high permeability material near the $z$ axis. Analogous results have been obtained in [7], see also [5], for different formulas for coefficients.

\section{Effect of the error}

With the geometry of Fig. 1 we have made computations of lens with different permeability of the polepieces. A rectangular fine mesh was used having constant step with 5 points between the symmetry plane and the polepiece face, and variable step elsewhere, allowing expansion by $5 \%$. The relative permeability $\mu_{\text {rel }}$ was changed between 10 and $10^{6}$, the relative permeability of the outside part of the circuit was $10^{6}$. Taking as a criterion of the error the percentage of the input excitation calculated as the integral of $H_{z}$ along the axis, the error is about $1.5 \%$. If we now close the circuit, and eventually put the polepiece up to the optical axis, the amount of lost excitation is increased dramatically - see Fig. 3.

\section{SOLUTION OF THE PROBLEM}

In the one-dimensional FEM formulation for $A(r)$, the integration method using the center values provides exact results both for $A$ and $r A$ [5]. Therefore we have tried to find an analogy in two dimensions that would provide correct integration



Fig. 3. Excitation on the axis for the lens from Fig. 1, and for the case of rod with $20 \mathrm{~mm}$ diameter instead of the polepieces, in dependence on the relative permeability of the rod or polepieces. of the term $A / r$ and that contains, in the case of a right angle triangle, the potentials of the nodal points on the side along the $r$ axis only. We have chosen for an average value of $B_{z}$ an expression

$$
B_{z}=\sum_{i} c_{i} A_{i} / \sum_{i} c_{i} r_{i}+\sum_{i} k_{i} A_{i} / \sum_{i} k_{i} l r_{i}
$$

while for $B_{r}=-g$ we have the usual form

$$
B_{r}=-\sum_{i} b_{i} A_{i} / \sum_{i} b_{i} z_{i}=-\sum_{i} b_{i} A_{i} / \sum_{i} c_{i} r_{i}
$$

with both expressions in the denominator of (6) being equal to $D$ in (2). The difference in the $r$ coordinates of the center of gravity of the triangle and the interval in the $r$ direction can be cancelled by using both possible divisions of the quadrilaterals into triangles. With the help of (5) and (6), new expressions of coefficients for FEM equations can be derived. They also give the same order of error $O\left(h^{2}\right)$, and differ from the other methods only by terms of higher order in mesh size $h$. On the cylindrical boundary, the local error in the new FEM approximation can be written as

$$
\begin{aligned}
A_{0}^{F E M}-A_{0}^{a n a l}= \\
=\frac{\mu_{0}\left(\rho_{1}-\rho_{2}\right) H_{z}}{48 r_{0} \rho_{1} \rho_{2}\left(\frac{1}{\mu_{1} \rho_{1}^{2}\left(r_{0}+r_{1}\right)}+\frac{1}{\mu_{2} \rho_{2}^{2}\left(r_{0}+r_{2}\right)}\right)}
\end{aligned}
$$

This error is no longer proportional to $A_{0}$, and it is independent of the relative permeability of the material on the axis. A further feature of the new method is that in rectangular meshes the coefficients of the FEM equations depend only on the nearest neighbors in $r$ and $z$ and not on the other corner potentials, as it also occurs in the method using potentials at the vertices and in the five point finite difference formulas. The computation effort for the coefficients is less than that for the previously chosen default method using the mid points of the sides.

\section{RESULTS}

The new method of coefficient computation has been implemented in the program LENS $[3,8]$ using the boundary condition $A=0$. Different methods for computation of coefficients can be realised by exchanging one subroutine where the evaluation of eq. (1) in each triangle is performed. The difference is then only in 15 lines of code. 
In the variant of LENS with the homogeneous Neumann boundary condition we can model cylindrical rods of infinite length with just 2 lines in the $z$ direction. The results obtained with different coefficient computation methods are illustrated in Fig. 4 for a cylindrical rod with permeability $\mu_{r e l}=1000$ with the s?me geometry as in the pot transformer of [5], i.e. rod radius $1 \mathrm{~mm}$, a coil with constant current density up to $r=8$ $\mathrm{mm}$, and a radial boundary at $r=12 \mathrm{~mm}$ at the position of outer shield. Constant stepsize was used between the axis and the line at $r=1 \mathrm{~mm}$, i.e. for $h_{r}=0.01 \mathrm{~mm}$ there were 100 mesh points in the first $1 \mathrm{~mm}$ radius, and a variable step mesh with expansion by $10 \%$ with 50 more points up to the outside radial boundary at $12 \mathrm{~mm}$. In this case, the new method of computation of coefficients gives very good agreement with the expected value of $B_{z}$ as given by the coil excitation.

Results obtained with the new method are also given in Fig. 3: the error in excitation is less than $0.1 \%$ for all values $\boldsymbol{\mu}_{\text {rel }}$ both for lens and rod examples.

\section{CONCLUSIONS}

The new method for the computation' of the coefficients of the FEM equations in an axisymmetric case for the vector



Fig. 4. The dependence of the relative axial flux density in a cylindrical rod of $1 \mathrm{~mm}$ radius with relative permeability $\mu_{\text {rel }}=1000$ [5], calculated by FEM with different coefficient computations, on the mesh step in the radial direction $h_{r}$. potential does not suffer from the inherent error of other methods when modelling cases similar to that one with high permeability cylindrical rod on the axis. We prefer our solution as compared with the solutions proposed in [5] and [6], as we still use shape functions linear in $r$ and $z$ coordinates. This is important, as the axisymmetric magnetic circuits are usually composed not only from cylindrical, but also from conical parts.

In order to decide if this new method should be implemented as a default coefficient method for FEM computations of magnetic lenses, further considerations are still necessary. In magnetic axisymmetric lenses the geometry is such, that the continuity of the normal component of the flux density at the material boundaries is crucial for the results.

\section{REFERENCES}

[1] E. Munro: "Computer-aided design of electron lenses by the finite element method", in "Image Processing and Computer Aided Design in Electron Optics", edited by P. W. Hawkes. London: Academic Press, 1973, pp. 284-323.

[2] E. Munro et al: "Field computation techniques in electron optics", IEEETransactions on Magnetics, Vol.MAG-26, No. 2, March 1990, pp. 1019-1022.

[3] B. Lencova, M. Lenc: "A finite element method for the computation of magnetic electron lenses", Scanning Electron Microscopy 1986/III, pp. 897-915. SEM Inc., AMF O'Hare, Chicago, 1986.

[4] K. Tahir, T.Mulvey: "Pitfalls in the calculation of the field distribution of magnetic electron lenses by the finite-element method", Nucl. Instr. Meth. in Phys. Research, Vol. A298, December 1990, pp. 383-388.

[5] J. B. M. Melissen, J. Simkin: "A new coordinate transform for the finite element solution of axisymmetric problems in magnetostatics", IEEE Transactions on Magnetics, Vol. MAG-26, No. 2, March 1990, pp. 391-394.

[6] A. Boglietti, M. Chiampi, D. Chiarabagtio, M. Tartaglia: "Finite element approximation in axisymmetrical domains", IEEE Transactions on Magnetics, Vol. MAG-26, No. 2, March 1990,pp. 395-398.

[7] J. B. M. Melissen, "Cylindrical coordinates in electromagnetic computations II, a one dimensional error sudy: $A$ versus $r A$ " Philips CPS Technical note Nr.010 UDR/MSW/089/HM092/hm (1989)

[8] B. Lencova, G. Wisselink: "Program package for the computation of lenses and deflectors", Nucl. Instr. Meth. in Phys. Research, Vol. A298, December 1990, pp. 56-66. 